\title{
CHI TIÊU CHÍNH PHỦ VÀ TĂNG TRƯởNG KINH TẾ TẠI MỘT SỐ QUỐC GIA ĐÔNG NAM Á
}

\author{
NGUYỄN THI MỸ LINH \\ Truờng Đại học Công nghiệp Thành phố Hồ Chí Minh \\ nguyenthimylinh@iuh.edu.vn
}

Tóm tắt. Bài nghiên cứu tập trung kiểm định sự tác động của chi tiêu chính phủ đến tăng trưởng kinh tế tại 05 quốc gia khu vực Đông Nam Á (ASEAN) gồm: Việt Nam, Thái Lan, Phillippine, Indonesia, Malaysia trong giai đoạn 2000-2016. Nghiên cứu sử dụng phương pháp định lượng hồi quy với dữ liệu bảng, cụ thể là phương pháp GMM (Generalized Method of Moment) nhằm khắc phục hiện tượng tự tương quan giữa các sai số, hiện tượng phương sai của sai số thay đổi và các vấn đề nội sinh tiềm ẩn. Kết quả nghiên cứu cho thấy chi tiêu chính phủ có tác động phi tuyến đến tốc độ tăng trưởng kinh tế tại các quốc gia nghiên cứu, cụ thể mức chi tiêu chính phủ tối ưu cho tăng trưởng kinh tế là $23,23 \%$ GDP. Đây là cơ sở để tác giả đưa ra một số gợi ý chính sách cho các nhà quản lý Việt Nam nhằm góp phần phát huy tác động tích cực của chi tiêu chính phủ đến tăng trưởng kinh tế.

Từ khóa. chi tiêu chính phủ; tăng trưởng kinh tế; Đông Nam Á; ASEAN.

\section{GOVERNMENT SPENDING AND ECONOMIC GROWTH IN SOUTHEAST ASIAN COUNTRIES}

\begin{abstract}
The study aims at examining the impacts of government spending on economic growth in five ASEAN countries including Vietnam, Thailand, Philippines, Indonesia, and Malaysia during the period between 2000 and 2016. This research employs the quantitative methodology of panel-data regression, particularly the Generalized Method of Moment (GMM) to deal with autocorrelation of residuals, problem of variance change and potential endogenous problems. The result reveals that government spending has non-linear impact on economic growth in countries under the study. More specifically, the optimal government spending for economic growth should be $23.23 \%$ percent of GDP. Accordingly, the authors suggest some policy recommendations to Vietnamese authorities in order to take advantages of positive influences of government spending on economic growth.
\end{abstract}

Keywords. government spending; economic growth; Southeast Asia; ASEAN.

\section{GIỚI THIỆ}

Các lý thuyết về tăng trưởng kinh tế có nhiều quan điểm về chi tiêu chính phủ, đối với các trường phái ủng hộ chi tiêu chính phủ lớn cho rằng việc chi tiêu chính phủ cung cấp các hàng hóa công sẽ làm tăng sức mua của người dân và thông qua đó đầy nhanh tăng trưởng kinh tế. Ngược lại, trường phái ủng hộ chi tiêu chính phủ nhỏ quan điểm là việc chi tiêu chính phủ sẽ chèn lấn khu vực tư phát triển và họ cho rằng khu vực tư sản xuất hiệu quả hơn, còn khu vực công kém hiệu quả, điều này dẫn đến kìm hãm tăng trưởng kinh tế. Nhìn chung, các lý thuyết kinh tế không khẳng định rõ ràng về tác động của chi tiêu chính phủ đến tăng trưởng kinh tế, nhưng đa phần các nhà kinh tế cũng cho rằng tăng qui mô chi tiêu của chính phủ có thể thúc đẩy tăng trưởng kinh tế, nhưng trong một số trường hợp việc cắt giảm chi tiêu của chính phủ sẽ tác động tích cực đến nền kinh tế.

Trong bối cảnh Việt Nam hiện nay, thâm hụt ngân sách đang ngày càng gia tăng, cắt giảm chi tiêu chính phủ đang được các nhà làm chính sách quan tâm và nổ lực thực hiện. Bài nghiên cứu với mong muốn có thêm bằng chứng thực nghiệm về tác động của chi tiêu chính phủ đến tăng trưởng kinh tế tại một số quốc gia trong ASEAN, trong đó có Việt Nam, từ đó đưa ra khuyến nghị phù hợp cho các nhà hoạch định chính sách tại Việt Nam. Bài nghiên cứu sử dụng phương pháp nghiên cứu định lượng với kỹ thuật phân tích hồi quy với dữ liệu bảng cho 05 quốc gia trong giai đoạn từ năm 2000-2016, để kiểm định chiều hướng 
của mối quan hệ này với nguồn dữ liệu thứ cấp thu thập được từ ngân hàng thế giới WB (World Bank) và Quỹ tiền tệ quốc tế IMF (International Monetary Fund).

\section{CƠ SỞ LÝ THUYẾT}

Keynes và các nhà kinh tế theo trường phái Keynes cho rằng chi tiêu chính phủ sẽ thúc đẩy tăng trưởng kinh tế thông qua tăng tổng cầu nền kinh tế. Tuy nhiên, khuyến cáo chi tiêu chính phủ không nên vượt quá $25 \%$ GDP, nếu tăng thêm qui mô chi tiêu chính phủ sẽ ảnh hưởng không tốt đến tăng trưởng kinh tế; ngược lại, cũng có những quan điểm cho rằng cắt giảm chi tiêu chính phủ làm giảm thâm hụt ngân sách, từ đó giảm lãi suất, tăng đầu tư và là động lực thúc đẩy tăng trưởng kinh tế.

Rahn (1986) với đường cong Rahn phản ánh mối quan hệ giữa quy mô chi tiêu chính phủ và tăng trưởng kinh tế. Theo đó, chi tiêu chính phủ đến ngưỡng tối ưu vừa đủ được phân bổ hết cho hàng hóa công cơ bản như cơ sở hạ tầng, bảo vệ pháp luật và quyền sở hữu sẽ thúc đẩy tăng trưởng kinh tế; ngược lại, qui mô chi tiêu vượt qua ngưỡng này sẽ tác động tiêu cực đến tăng trưởng kinh tế. Mức chi tiêu tối ưu với tăng trưởng kinh tế được khuyến cáo ở mức $15 \%$ đến $25 \%$ GDP.

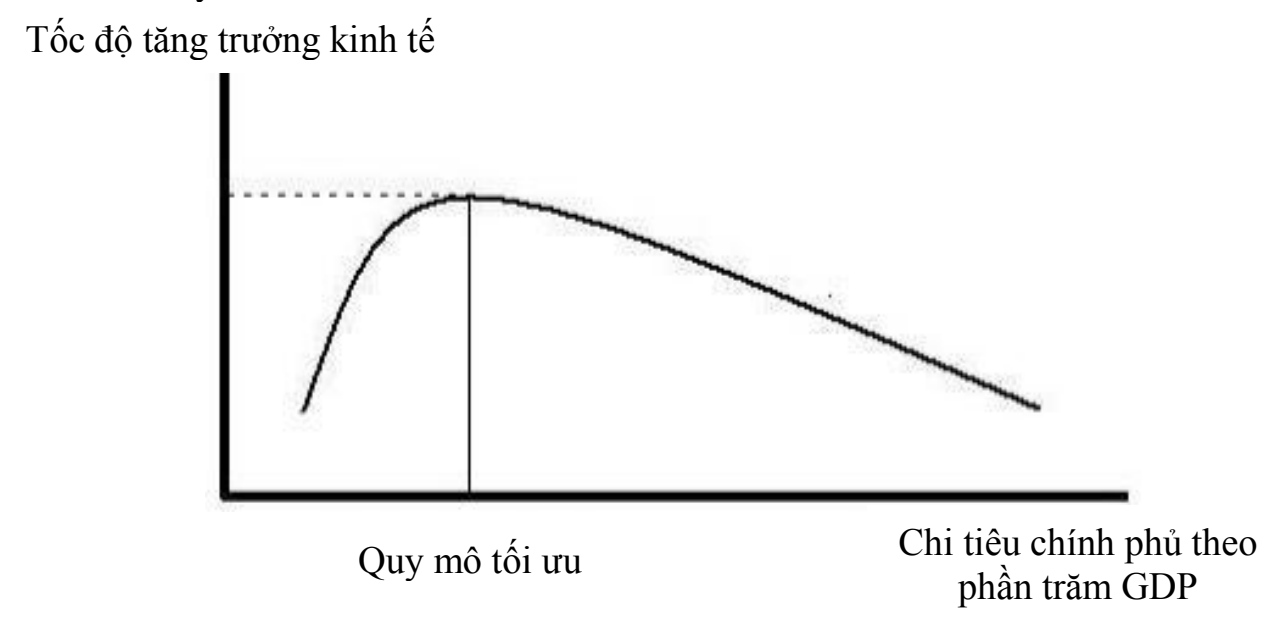

Hình 1. Đuòng cong Rahn

Việc giảm tăng trưởng kinh tế trên đường cong Rahn được giải thích là: (i) khi tăng qui mô chi tiêu chính phủ vượt quá sức chịu đựng của nền kinh tế đều cần có nguồn tài trợ từ tăng thuế hoặc vay nợ, điều này làm giảm tiết kiệm, giảm nguồn vốn đầu tư tư nhân, tăng lãi suất; (ii) chi tiêu chính phủ sẽ chèn lấn khu vực đầu tư tư nhân, làm giảm tính cạnh tranh của khu vực tư nhân, trong khi đó quyết định chi tiêu chính phủ bị chi phối bởi các lực lượng chính trị và thường là kém hiệu quả hơn khu vực tư nhân; (iii) một số chương trình chi tiêu của chính phủ không khuyến khích gia tăng sản xuất như bảo hiểm thất nghiệp làm giảm động cơ tìm việc làm, trợ cấp hưu trí hay trợ cấp cho giáo dục không khuyến khích tiết kiệm;... các điều này sẽ dẫn đến hệ quả tiêu cực đối với tăng trưởng kinh tế.

Hàm ý tương tự như Rahn (1986), Barro (1990) kết luận rằng, việc tăng thuế để bù đắp chi tiêu của chính phủ chỉ tác động tích cực đến tăng trưởng kinh tế khi tác động tích cực của việc tăng chi tiêu cao hơn tác động tiêu cực của tăng thuế.

Trên cơ sở nghiên cứu của Barro (1990), Devarajan, Swaroop, và Zou (1996) chỉ ra rằng sự điều chỉnh các thành phần chi tiêu chính phủ có tác động đến tăng trưởng kinh tế, theo đó, tăng chi tiêu đối với các khoản mục có hiệu quả và giảm chi tiêu đối với các khoản mục không hiệu quả sẽ cải thiện tăng trưởng kinh tế. Một nghiên cứu khác của Davoodi và Zou (1998) dựa trên cơ sở nghiên cứu của Barro (1990), Devarajan, Swaroop, và Zou (1996) dưới góc nhìn của phân cấp chi tiêu ngân sách, nghiên cứu chỉ ra rằng, với tổng mức chi tiêu là không đổi, thay đổi tỷ trọng chi giữa các cấp ngân sách sẽ thúc đẩy tăng trương kinh tế nếu phân bổ hiện tại là chưa tối ưu.

Một số các nghiên cứu thực nghiệm sau đây là minh chứng bổ sung cho lý thuyết về tăng trưởng kinh tế dưới tác động của chi tiêu chính phủ.

Grier \& Tullock, (1989) với dữ liệu nghiên cứu trên cỡ mẫu 113 quốc gia, gồm 24 nước OECD và 89 nước khác, chiều dài dữ liệu nghiên cứu là 30 năm cho các nước $\mathrm{OECD}$ và 20 năm cho các nước còn lại, 
kết quả là bằng chứng thực nghiệm cho tác động tiêu cực giữa tăng trưởng chi tiêu chính phủ và tăng trưởng kinh tế, cụ thể tại các nước OECD, Châu Phi, Trung và Nam Mỹ.

Ighodaro \& Oriakhi (2010) khi nghiên cứu dữ liệu của Nigeria trong giai đoạn 1961 - 2007 đã cho rằng có sự tác động cùng chiều của chi tiêu chính phủ đến tăng trưởng kinh tế.

Cùng thời điểm này, Nurudeen \& Usman (2010) đã nghiên cứu dữ liệu trong giai đoạn 1977 - 2007 tại Nigeria đã tìm thấy tác động của chi tiêu chính phủ đến tăng trưởng kinh tế, cụ thể: tổng chi đầu tư của chính phủ, tổng chi thường xuyên và chi tiêu chính phủ cho giáo dục có ảnh hưởng ngược chiều đến tăng trưởng kinh tế; trong khi đó, chi tiêu cho vận tải, truyền thông và sức khoẻ ảnh hưởng cùng chiều đến tăng trưởng kinh tế.

Cũng tại Nigeria, Okoro (2013) đã nghiên cứu sự tác động của chi tiêu chính phủ đến tăng trưởng kinh tế trong giai đoạn 1980-2011. Kết quả nghiên cứu cho thấy có sự tác động cùng chiều của chi tiêu chính phủ đến tăng trưởng kinh tế. Cho kêt quả tương tự Okoro (2013), Asghar \& các cộng sự (2011) khi nghiên cứu dữ liệu của Pakistan trong giai đoạn 1974-2008 đã cho rằng chi tiêu chính phủ cũng có tác động cùng chiều đến tăng trưởng kinh tế.

Yasin (2003) đã nghiên cứu dữ liệu của các nước thuộc tiểu vùng Sahara (Châu Phi) trong giai đoạn 1987-1997. Kết quả nghiên cứu cho thấy chi tiêu của chính phủ, độ mở thương mại và chi tiêu hộ gia đình có ảnh hưởng cùng chiều đến tăng trưởng kinh tế.

Gần đây, Mushtaq \& các cộng sự (2014) đã sử dụng dữ liệu của 08 quốc gia trong giai đoạn 1995 2011 và cho rằng chi tiêu chính phủ, kim ngạch xuất khẩu và đầu tư tư nhân trong nước tác động cùng chiều đến tăng trưởng kinh tế. Trong khi đó, kim ngạch nhập khẩu tác động ngược đến tăng trưởng kinh tế.

Một số các nghiên cứu khác như Plossner (1992), Levine và Renelt (1992), Florin-Marius Pavelescu (2008), Jhingan (2006), Ainabor và cộng sự (2014) đã kết luận Tỷ lệ tích lũy tài sản có tác động đến tăng trưởng kinh tế; bên cạnh đó, chi tiêu hộ gia đình ảnh hưởng đến tăng trưởng kinh tế là kết quả nghiên cứu thực nghiệm của Khan và Kumar (1997), OECD (2009), Guisan (2004); ngoài Tỷ lệ tích lũy tài sản, chi tiêu hộ gia đình, thì độ mở thương mại cũng là yếu tố quan trọng có ý nghĩa với tăng trưởng kinh tế theo Prabirjit (2007), Frankel và Romer (1999), Dollar (1992), Sachs và Warner (1995).

\section{PHƯƠNG PHÁP NGHIÊN CÚU VÀ DŨ LIỆU NGHIÊN CƯU}

\subsection{Phương pháp nghiên cứu}

Nghiên cứu sử dụng phương pháp định lượng với dữ liệu bảng thông qua kỹ thuật phân tích hồi quy tuyến tính đa biến để lượng hóa sự tác động của các biến độc lập lên biến phụ thuộc trong mô hình. Trước tiên, nghiên cứu hồi quy theo 3 phương pháp thông thường trên dữ liệu bảng: hồi quy theo phương pháp bình phương nhỏ nhất (Pooled OLS); hồi quy ảnh hưởng cố định (FEM) và hồi quy ảnh hưởng ngẫu nhiên (REM). Để lựa chọn phương pháp hồi quy nào là phù hợp nhất trong ba phương pháp trên, các kiểm định được sử dụng là: kiểm định F-test và kiểm định Breusch and Pagan Lagrangian Multiplier (Breush \& Pagan, 1979). Kiểm định F-test để chọn lựa giữa mô hình Pooled OLS và mô hình FEM. Kiểm định Hausman để biết được nên chọn mô hình FEM hay mô hình REM.

Tiếp theo, bài nghiên cứu tiến hành kiểm định hiện tượng tự tương quan và hiện tượng phương sai của sai số thay đổi. Sau đó, bài nghiên cứu sử dụng phương pháp hồi quy GMM (Generalized Method of Moment) để giải quyết các vấn đề nội sinh tiềm ẩn, hiện tượng tự tương quan giữa các sai số và hiện tượng phương sai của sai số thay đổi (Doytch \& Uctum, 2011). Theo Driffill \& các cộng sự (1998), phương pháp hồi quy GMM tốt hơn các phương pháp hồi quy thông thường trên dữ liệu bảng trong việc kiểm tra sự chuyển động của các biến tài chính. Trong phần này, tác giả sẽ sử dụng kiểm định Sargan nhằm xác định tính chất phù hợp của các biến công cụ trong ước lượng GMM. Kiểm định Sargan với giả thuyết $\mathrm{H}_{0}$ : biến công cụ là ngoại sinh, nghĩa là biến công cụ không tương quan với sai số của mô hình. Để kiểm định hiện tượng tự tương quan, bài nghiên cứu sử dụng kiểm định Arellano-Bond với giả thuyết $\mathrm{H}_{0}$ : không có hiện tượng tự tương quan.

\subsection{Dũ̃ liệu nghiên cứu}

Bài nghiên cứu sử dụng dữ liệu thứ cấp của Ngân hàng thế giới (WB) và Quỹ tiền tệ quốc tế (IMF) cho 05 quốc gia trong ASEAN: Việt Nam, Thái Lan, Phillippine, Indonesia, Malaysia trong giai đoạn 20002016. Sau khi dữ liệu được thu thập, tác giả thực hiện bước tiếp theo là tính toán các biến dựa trên số liệu thu thập được để tiến hành các bước kiểm định và phân tích hồi quy. 


\subsection{Mô hình nghiên cứu}

Căn cứ vào kết quả của các nghiên cứu thực nghiệm được trình bày ở mục 2 , tác giả xây dựng mô hình nghiên cứu với biến phụ thuộc là tốc độ tăng trưởng kinh tế (GDP), biến giải thích trong mô hình là chi tiêu chính phủ. Nhằm kiểm soát sự tác động của một số biến số khác đối với tốc độ tăng trưởng kinh tế, tác giả đưa thêm các biến: tỷ lệ tích lũy tài sản, chi tiêu hộ gia đình và độ mở thương mại vào mô hình nghiên cứu. Ngoài ra, tác giả tiến hành bổ sung thêm biến độc lập chi tiêu chính phủ bình phương nhằm kiểm định tác động phi tuyến của chi tiêu chính phủ đến tăng trưởng kinh tế. Việc đưa thêm biến độc lập chi tiêu chính phủ bình phương được tác giả căn cứ vào lý thuyết đường cong Rahn, lý thuyết này được Rahn đưa ra vào năm 1986, tuy nhiên tác giả chưa tìm thấy nghiên cứu thực nghiệm nào gần đây tiến hành kiểm định phi tuyến dưới dạng mô hình nghiên cứu định lượng, do đó tác giả đưa vào mô hình để nghiên cứu, đồng thời tạo tính mới cho bài nghiên cứu này.

Vậy, mô hình nghiên cứu dự kiến có phương trình như sau:

$$
\mathrm{GDP}_{\mathrm{it}}=\beta_{0}+\beta_{1} \mathrm{GOV}_{\mathrm{it}}+\beta_{2} \mathrm{GOV}^{2}{ }_{\mathrm{it}}+\beta_{3} \mathrm{ASSET}_{\mathrm{it}}+\beta_{4} \mathrm{FAM}_{\mathrm{it}}+\beta_{5} \mathrm{OPEN}_{\mathrm{it}}+\varepsilon_{\mathrm{it}}
$$

Trong đó: i: quốc gia thứ i (i = 1-5); t: năm (từ 2000-2016)

Bảng 1. Ký hiệu mã biến, tên biến, đo luờng biến và nhũng nghiên cứu truớc liên quan đến các biến trong mô hìh

\begin{tabular}{|c|c|c|c|}
\hline Mã biến & Tên biến & Đo lường biến & Các nghiên cứu trước \\
\hline \multicolumn{4}{|c|}{ Biến phụ thuộc } \\
\hline $\mathrm{GDP}_{\text {it }}$ & $\begin{array}{l}\text { Tăng trưởng kinh } \\
\text { tế }\end{array}$ & $\begin{array}{l}\text { Tốc độ tăng trưởng kinh tế hàng } \\
\text { năm }\end{array}$ & \\
\hline \multicolumn{4}{|c|}{ Biến độc lập } \\
\hline $\mathrm{GOV}_{\text {it }}$ & $\begin{array}{l}\text { Chi tiêu chính } \\
\text { phủ }\end{array}$ & $\begin{array}{l}\text { Tỷ lệ chi tiêu chính phủ so với } \\
\text { GDP (\% GDP) }\end{array}$ & $\begin{array}{l}\text { Grier \& Tullock, (1989); Asghar \& các } \\
\text { cộng sự (2011); Ighodaro \& Oriakhi } \\
\text { (2010); Mushtaq \& các cộng sự (2014); } \\
\text { Nurudeen \& Usman (2010); Okoro } \\
\text { (2013); Yasin (2003) }\end{array}$ \\
\hline $\mathrm{GOV}^{2}{ }_{\text {it }}$ & $\begin{array}{l}\text { Chi tiêu chính } \\
\text { phủ bình phương }\end{array}$ & $\begin{array}{l}\text { (Tỷ lệ chi tiêu chính phủ so với } \\
\text { GDP) }\end{array}$ & Xây dựng mới (kiểm định phi tuyến) \\
\hline \multicolumn{4}{|c|}{ Biến kiểm soát } \\
\hline $\mathrm{ASSET}_{\text {it }}$ & $\begin{array}{l}\text { Tỷ lệ tích lũy tài } \\
\text { sản }\end{array}$ & $\begin{array}{l}\text { Tỷ lệ tích lũy tài sản hàng năm } \\
\text { so với GDP (\% GDP)= các } \\
\text { khoản đầu tư cho tài sản cố định } \\
\text { gộp của nền kinh tế }{ }^{1} \text { (chính phủ } \\
\text { và tư nhân) + thay đồi ròng trong } \\
\text { giá trị hàng tồn kho }{ }^{2}\end{array}$ & $\begin{array}{l}\text { Mushtaq \& các cộng sự (2014); } \\
\text { Plossner (1992); Levine và Renelt } \\
\text { (1992); Florin-Marius Pavelescu (2008). }\end{array}$ \\
\hline $\mathrm{FAM}_{\mathrm{it}}$ & $\begin{array}{l}\text { Chi tiêu hộ gia } \\
\text { đình }\end{array}$ & $\begin{array}{l}\text { Tốc độ tăng chi tiêu hộ gia đình } \\
\text { hàng năm (\% GDP) }\end{array}$ & $\begin{array}{l}\text { Yasin (2003); Khan và Kumar (1997); } \\
\text { OECD (2009); Guisan (2004) }\end{array}$ \\
\hline $\mathrm{OPEN}_{\mathrm{it}}$ & $\begin{array}{l}\text { Độ mở thương } \\
\text { mại }\end{array}$ & $\begin{array}{l}\text { Tốc độ tăng tổng kim ngạch xuất } \\
\text { khẩu, nhập khẩu hàng hóa và } \\
\text { dịch vụ hàng năm }(\%)\end{array}$ & $\begin{array}{l}\text { Mushtaq \& các cộng sự (2014); Yasin } \\
\text { (2003); Prabirjit (2007); Frankel và } \\
\text { Romer (1999); Dollar (1992); Sachs và } \\
\text { Warner (1995) }\end{array}$ \\
\hline
\end{tabular}

Nguồn: Tổng hợp của tác giả

\section{KẾT QUẢ NGHIÊN CỨU}

\subsection{Thống kê mô tả}

Dữ liệu nghiên cứu được thu thập từ WB và IMF cho 05 quốc gia trong ASEAN, giai đoạn 2000-2016 với các biến số được mô tả trong bảng 2 sau đây:

\footnotetext{
${ }^{1}$ Các khoản đầu tu bổ sung cho tài sản cố định nhu đầu tư cho cải tạo đất; đầu tu cho nhà xuởng, máy móc, truờng hoc, bệnh viện, nhà ở tu nhân, các tòa nhà thuơng mại và công nghiệp

${ }^{2}$ Hàng tồn kho là hàng hóa của doanh nghiệp được dụ trũ cho hoạt kinh doanh của doanh nghiẹp.
} 
Bảng 2. Thống kê mô tả các biến

\begin{tabular}{|c|c|c|c|c|c|}
\hline Biến & Số quan sát & Nhỏ nhất & Trung bình & Cao nhất & Độ lệch chuẩn \\
\hline GDP $_{\text {it }}$ & 85 & $-1,514$ & 5,2368 & 8,671 & 1,925938 \\
\hline GOV $_{\text {it }}$ & 85 & 15,303 & 22,60499 & 31,606 & 4,337167 \\
\hline ASSET $_{\text {it }}$ & 85 & $-25,1248$ & 7,048845 & 31,61425 & 10,35836 \\
\hline FAM $_{\mathrm{it}}$ & 85 & $-1,25461$ & 5,366169 & 13,02843 & 2,441415 \\
\hline OPEN $_{\mathrm{it}}$ & 85 & $-18,629$ & 4,786014 & 25,81378 & 8,655016 \\
\hline
\end{tabular}

Nguồn: Kết quả phân tích của tác giả

Tăng trưởng GDP thấp nhất và cao nhất trong các quốc gia nghiên cứu đều thuộc về Malaysia vào năm 2009 và năm 2000. Tỷ lệ chi tiêu của chính phủ Indonesia thấp nhất vào năm 2000 , trong khi đó tỷ lệ chi tiêu của chính phủ Việt Nam cao nhất vào năm 2009. Tốc độ tích lũy tài sản thấp nhất thuộc về Thái Lan vào năm 2009 và và cao nhất là Philippine năm 2010. Thái Lan là quốc gia có tốc độ tăng chi tiêu của hộ gia đình thấp nhất vào năm 2009, ngược lại, chỉ số này cao nhất thuộc về Malaysia vào năm 2000 . Tổng kim ngạch xuất nhập khẩu của Indonesia giảm mạnh nhất vào năm 2009, ngược lại, Thái Lan dẫn đầu các quốc gia nghiên cứu về tốc độ tăng chỉ số này vào năm 2000 .

\subsection{Kết quả hồi quy}

Nghiên cứu áp dụng các phương pháp hồi quy trên dữ liệu bảng, bao gồm: hồi quy tuyến tính theo phương pháp bình phương nhỏ nhất tồng quát (Pooled Regression - Pooled OLS); hồi quy ảnh hưởng cố định (Fixed effects model - FEM) và hồi quy ảnh hưởng ngẫu nhiên (Random effects model - REM). Kết quả nghiên cứu cho thấy phương pháp hồi quy FEM tỏ ra phù hợp hơn do kiểm định $F(4,75)=4,58$ có ý nghĩa thống kê ở mức ý nghĩa 1\%, kiềm định Hausman chi2(5) = 39,52 có ý nghĩa thống kê ở mức ý nghĩa $1 \%$. Tuy nhiên, mô hình nghiên cứu có hiện tượng tự tương quan giữa các sai số và hiện tượng phương sai của sai số thay đổi, các hiện tượng này có thể được kiểm soát bằng phương pháp GMM (Generalized Method of Moment) nhằm đảm bảo ước lượng thu được vững và hiệu quả, ngoài ra phương pháp này còn giải quyết được các vấn đề nội sinh tiềm ẩn (Doytch \& Uctum, 2011). Kết quả các mô hình nghiên cứu như Bảng 3.

Bảng 3. Kết quả phuơng sai của sai số thay đổi và tụ tương quan

\begin{tabular}{|c|c|}
\hline Kiểm định phương sai của sai số thay đổi & Kiểm định tự tương quan giữa các sai số \\
\hline White's test & Wooldridge test \\
\hline chi2 $2(19)=48,25$ & $\mathrm{~F}(1,4)=31,662$ \\
Prob $>\operatorname{chi} 2=0,0002^{* * *}$ & Prob $>\mathrm{F}=0,0049^{* * *}$ \\
\hline
\end{tabular}

Ghi chú: ${ }^{* * *}$ có ý nghĩa turong úng ở múc $1 \%$ Nguồn: Kết quả phân tích của tác giả

Kiểm định White cho thấy mô hình có hiện tượng phương sai của sai số thay đổi với mức ý nghĩa 1\%. Kiểm định Wooldridge cho thấy mô hình có hiện tượng tự tương quan giữa các sai số với mức ý nghĩa 1\%.

Với biến phụ thuộc là tăng trưởng kinh tế (GDP $\left.{ }_{i t}\right)$, sau khi dùng phương pháp GMM để giải quyết các vấn đề nội sinh tiềm ẩn, hiện tượng tự tương quan giữa các sai số và hiện tượng phương sai của sai số thay đổi, ta có kết quả nghiên cứu như Bảng 4.

Kết quả kiểm định Hansen của các mô hình trên khẳng định rằng các công cụ có thể coi là hợp lệ vì chấp nhận giả thuyết $\mathrm{H}_{0}\left(\mathrm{H}_{0}\right.$ : biến công cụ không tương quan với sai số của mô hình). Kiểm kiểm định Arellano-Bond cho thấy chấp nhận giả thuyết $\mathrm{H}_{0}$, tức là mô hình khá tốt do không có hiện tượng tự tương quan giữa các sai số.

Với mức ý nghĩa $5 \%$, biến độc lập chi tiêu chính phủ $\left(\mathrm{GOV}_{\text {it }}\right)$ tác động cùng chiều và chi tiêu chính phủ bình phương $\left(\mathrm{GOV}^{2}{ }_{\mathrm{it}}\right)$ tác động ngược chiều đến tăng trưởng kinh tế $\left(\mathrm{GDP}_{\mathrm{it}}\right)$. Điều này phản ánh rằng có tác động phi tuyến của chi tiêu chính phủ đến tăng trưởng kinh tế. Với biến độc lập chi tiêu chính phủ $\left(\mathrm{GOV}_{\mathrm{it}}\right)$, kết quả này tương đồng với các nghiên cứu của Asghar \& các cộng sự (2011), Ighodaro \& Oriakhi (2010), Mushtaq \& các cộng sự (2014), Nurudeen \& Usman (2010), Okoro (2013), Yasin (2003). Đối với biến chi tiêu chính phủ bình phương $\left(\mathrm{GOV}^{2}{ }^{2}\right)$, biến này tác động ngược chiều và có ý nghĩa thống kê đến tăng trưởng kinh tế $\left(\mathrm{GDP}_{\mathrm{it}}\right)$ phản ánh tính mới của nghiên cứu. Điều này có nghĩa là tăng chi tiêu của chính 
phủ có thể thúc đẩy tăng trưởng kinh tế, tuy nhiên chi tiêu của chính phủ vượt qua ngưỡng tối ưu sẽ tác động tiêu cực đến tăng trưởng kinh tế, phù hợp với lý thuyết của Keynes, đường cong Rahn (1986), và nghiên cứu của Barro (1990).

Bảng 4. Kết quả mô hình nghiên cưu

\begin{tabular}{|c|c|c|c|c|}
\hline \multirow{2}{*}{ GDP $_{\text {it }}$} & \multicolumn{4}{|c|}{ Hệ số hồi quy } \\
\hline & Pooled OLS & FEM & REM & GMM \\
\hline $\mathrm{GOV}_{\text {it }}$ & $-0,3850582$ & 0,1293934 & $-0,3850582$ & $3,532339^{* *}$ \\
\hline $\mathrm{GOV}^{2}{ }_{\mathrm{it}}$ & 0,0068149 & $-0,0060648$ & 0,0068149 & $-0,0760218^{* *}$ \\
\hline $\mathrm{ASSET}_{\text {it }}$ & $0,0441677^{\text {*** }}$ & $0,0439579^{* * *}$ & $0,0441677^{\text {*** }}$ & $0,0699106^{* * *}$ \\
\hline FAM $_{\text {it }}$ & $0,4200152^{* * *}$ & $0,4040723^{* * *}$ & $0,4200152^{* * *}$ & $0,3063806^{\text {**** }}$ \\
\hline $\mathrm{OPEN}_{\mathrm{it}}$ & $0,0471356^{* *}$ & 0,0280222 & $0,0471356^{* *}$ & 0,0201624 \\
\hline Hằng số & 7,541228 & 2,911346 & 7,541228 & $-36,40762^{*}$ \\
\hline Mức ý nghĩa & $\begin{array}{c}\mathrm{F}(5,79)=21,66 \\
\text { Prob }>\mathrm{F}= \\
0,0000^{* * *}\end{array}$ & $\begin{array}{c}\mathrm{F}(5,75) \\
21,63 \\
\text { Prob }>\mathrm{F} \\
0,0000^{* * *}\end{array}$ & $\begin{array}{l}\text { Wald chi2(5) }= \\
\quad 108,31= \\
\text { Prob }>\text { chi2 } \\
0,0000^{* * * *}\end{array}$ & $\begin{array}{c}\text { Wald chi2(4) }= \\
82,53 \\
\text { Prob }>\text { chi } 2= \\
0,000^{* * * *}\end{array}$ \\
\hline $\mathrm{R}^{2}$ & $57,82 \%$ & $51,33 \%$ & $57,82 \%$ & \\
\hline \multicolumn{4}{|c|}{ Kiểm định Arellano-Bond AR(2) } & $\operatorname{Pr}>\mathrm{z}=0,220$ \\
\hline \multicolumn{4}{|c|}{ Kiểm định Sargan } & $\begin{array}{c}\text { Prob }>\text { chi } 2= \\
0,117\end{array}$ \\
\hline
\end{tabular}

Ghi chú: ***, **, *có ý nghĩa tuơng úng ỏ múc 1\%, 5\%, $10 \%$ Nguồn: Kết quả phân tích của tác giả

Ngoài ra, tác giả còn tìm thấy tác động cùng chiều của biến kiểm soát tỷ lệ tích lũy tài sản $\left(\right.$ ASSET $\left._{\text {it }}\right)$ và chi tiêu hộ gia đình $\left(\mathrm{FAM}_{\mathrm{it}}\right)$ đến tăng trưởng kinh tế $\left(\mathrm{GDP}_{\mathrm{it}}\right)$ với mức ý nghĩa $1 \%$. Điều này cho thấy khi tăng tỷ lệ tích lũy tài sản và chi tiêu hộ gia đình có thể thúc đẩy tăng trưởng kinh tế.

Kết quả mô hình nghiên cứu có phương trình như sau:

\section{GDP $_{\text {it }}=-36,40762+3,532339$ GOV $_{\text {it }}-0,0760218$ GOV $^{2}{ }_{i t}+0,0699106$ ASSET $_{\text {it }}+0,3063806$ FAM $_{\text {it }}+$ \\ $\varepsilon_{\text {it }}$}

Với kết quả này, tác giả tiến hành khảo sát hàm số đối với phương trình bậc 2 trên theo GOV và được quy mô chi tiêu chính phủ tối ưu ở mức $23,23 \%$ GDP. Vậy, mức chi tiêu chính phủ được tác giả khuyến cáo ở mức 23,23\% GDP.

\section{KẾT LUẬN VÀ KHUYÉN NGH!}

Bài nghiên cứu tập trung kiểm định sự tác động của chi tiêu chính phủ đến tăng trưởng kinh tế tại 05 quốc gia khu vực Đông Nam Á (ASEAN) gồm: Việt Nam, Thái Lan, Phillippine, Indonesia, Malaysia trong giai đoạn 2000-2016. Tác giả đã áp dụng phương pháp hồi quy GMM để giải quyết các vấn đề nội sinh tiềm ẩn, hiện tượng tự tương quan giữa các sai số và hiện tượng phương sai của sai số thay đổi nhằm đảm bảo ước lượng thu được vững và hiệu quả. Kết quả nghiên cứu cho thấy tăng trưởng kinh tế $\left(\mathrm{GDP}_{\mathrm{it}}\right)$ bị tác động cùng chiều bởi chi tiêu chính phủ $\left(\mathrm{GOV}_{\text {it }}\right)$. Trong khi đó, chi tiêu chính phủ bình phương $\left(\mathrm{GOV}^{2}{ }_{i t}\right)$ tác động ngược chiều đến tăng trưởng kinh tế $\left(\mathrm{GDP}_{\mathrm{it}}\right)$. Ngoài ra, tác giả cũng tìm thấy tác động của tỷ lệ tích lũy tài sản $\left(\mathrm{ASSET}_{\mathrm{it}}\right)$ và chi tiêu hộ gia đình $\left(\mathrm{FAM}_{\mathrm{it}}\right)$ đến tăng trưởng kinh tế $\left(\mathrm{GDP}_{\mathrm{it}}\right)$. Với kết quả này, tác giả khuyến cáo chi tiêu chính phủ tối ưu ở mức 23,23\% GDP. Đây cũng là cơ sở để tác giả đưa ra một số gợi ý chính sách cho các nhà quản lý Việt Nam nhằm góp phần phát huy tác động tích cực của chi tiêu chính phủ đến tăng trưởng kinh tế.

Theo thống kê từ IMF trong giai đoạn nghiên cứu, chi tiêu chính phủ Việt Nam ở mức trung bình 27,60\%/năm, khá cao so với các quốc gia nghiên cứu, mức chi tiêu chính phủ trung bình của các quốc gia nghiên cứu là $22,60 \%$, đồng thời cao hơn mức chi tiêu khuyến cáo tối ưu từ kết quả nghiên cứu của tác giả $(23,23 \%)$. Như vậy, xét về mặt lý thuyết, Việt Nam cần giảm chi tiêu trong thời gian tới để đạt ngưỡng tối ưu cho tăng trưởng kinh tế. 


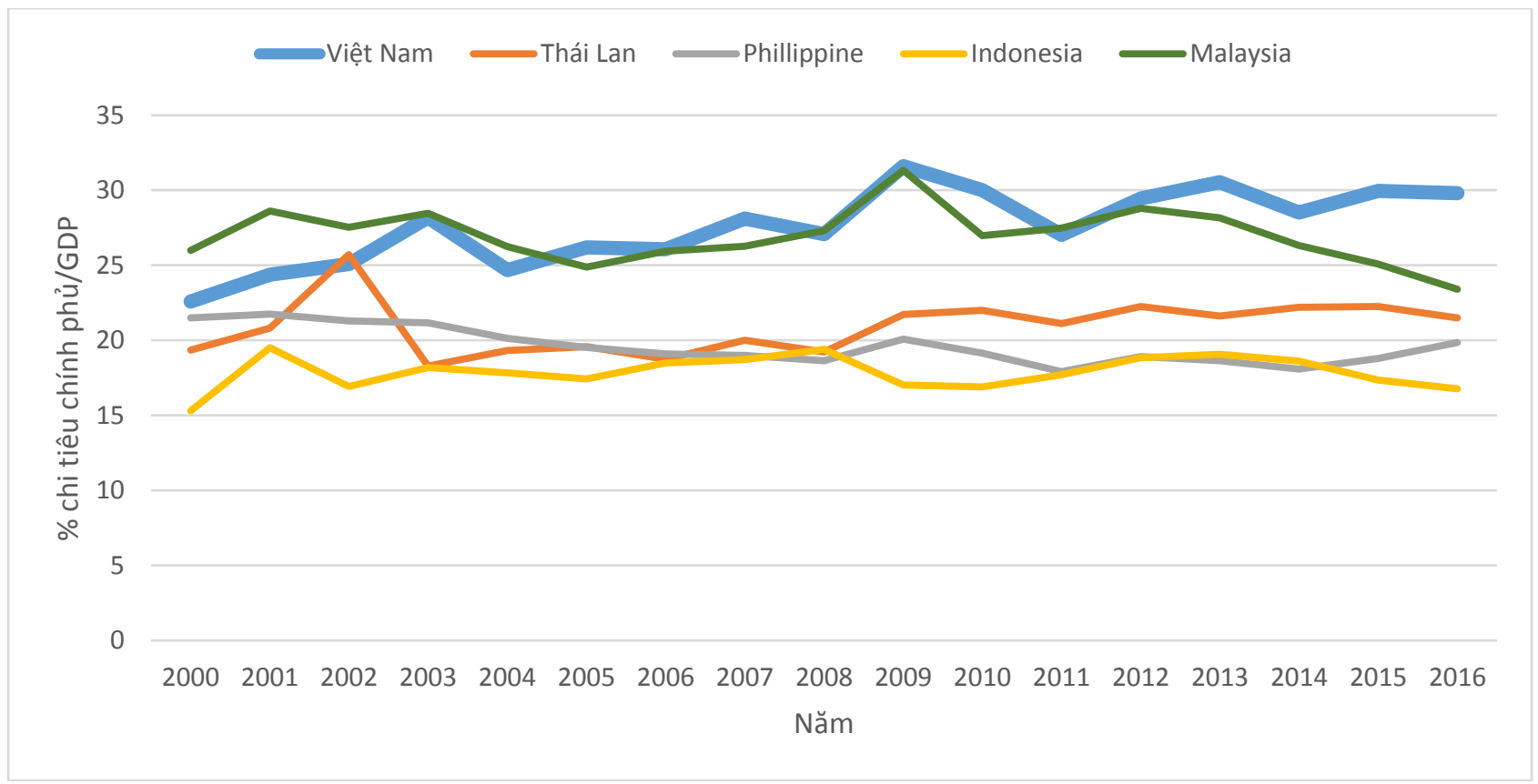

Hình 2. So sánh quy mô chi tiêu chính phủ giữa các quốc gia nghiên cứu

Hiện nay, theo đánh giá chung của nhiều nghiên cứu cho thấy chi tiêu công tại Việt Nam kém hiệu quả, vấn đề tiêu cực và thiếu trách nhiệm trong chi tiêu cũng được dư luận khá quan tâm trong thời gian qua. Với áp lực trả nợ nước ngoài cùng với việc cắt giảm mạnh mẽ các khoản vay ưu đãi do Việt Nam không còn nằm trong nhóm nước được nhận vay ưu đãi từ các tổ chức quốc tế, vấn đề cân đối nguồn lực tài chính để hạn chế thâm hụt ngân sách, đảm bảo trần nợ công đang được các nhà làm chính sách đặc biệt quan tâm. Như tác giả giới thiệu ở phần đầu của bài nghiên cứu này, giải pháp cắt giảm chi tiêu chính phủ đang được các nhà làm chính sách Việt Nam nổ lực thực hiện. Với kêt quả của bài nghiên cứu này, tác giả có thêm bằng chứng thực nghiệm ủng hộ chính sách cắt giảm chi tiêu chính phủ và đồng thời đưa ra một số khuyến nghị sau:

- Hạn chế đầu tư công dàn trải, cải cách và nâng cao hiệu quả của đầu tư công, tránh thất thoát, lãng phí.

- Giảm các khoản chi tiêu thường xuyên mà chủ yếu thông qua việc tinh giảm biên chế cán bộ hưởng lương từ ngân sách Nhà nước, hiện nay chi lương chiếm đến $2 / 3$ tổng chi thường xuyên là quá lớn.

- Cần minh bạch trong chi tiêu chính phủ, cần kiểm soát chặt chẽ các khoản chi tiêu của chính phủ và các bộ, ngành nhằm giảm gánh nặng cho ngân sách.

- Tăng các khoản chi có tác động tích cực lớn hơn với tăng trưởng kinh tế như giao thông vận tải, giáo dục và đào tạo; đồng thời, giảm chi tiêu thường xuyên và thay vào đó là chi cho đầu tư phát triển (Phạm Thế Anh, 2008). Song, tùy theo mục tiêu kinh tế xã hội chính phủ đang theo đuổi mà lựa chọn cơ cấu chi cho phù hợp trong từng giai đoạn.

\section{TÀI LIỆU THAM KHẢO}

[1] Ainabor, A.E, Shuaib I.M, Kadiri, A.K. (2014). Impact of Capital Formation on the Growth of Nigerian Economy 1960-2010: Vector Error Correction Model (VECM), School of Business Studies, Readings in Management and Social Studies, 1(1): 132-154

[2] Asghar, N., Azim, P., Rehman, H (2011). Impact of Government Spending in Social Sectors on Economic Growth: A Case Study of Pakistan, Journal Of Business \& Economics, Vol. 3 No. 2 (July-Dec 2011) pp.214234.

[3] Barro, R. (1990), "Government spending in a simple model of endogenous growth", Journal of Political Economy 98, 103-25 
[4] Breusch, S., Pagan, R (1979). A Simple Test for Heteroskedasticity and Random Coefficient Variation. Econometrica. 47 (5): 1287-1294.

[5] Davoodi, H., and Zou, H., (1998), "Fiscal Decentralization and Economic Growth: A Cross-Country Study", Journal of Urban Economics, 43, 244-257.

[6] Devarajan, S., Swaroop, V., and Zou, H., (1996), "The composition of public expenditure and economic growth", Journal of Monetary Economics, 37, 313-44.

[7] Dollar, D. (1992). Outward-oriented developing economies really do grow more rapidly: Evidence from 95 LDCs, 1976-85. EconomicDevelopmentand Cultural Change1992:523-544.

[8] Doytch, N., \& Uctum, M (2011). Does the worldwide shift of FDI from manufacturing to services accelerate economic growth? A GMM estimation study, Journal of International Money and Finance, 30(3), 410-427.

[9] Driffill, J., Psaradakis, Z., \& Sola, M (1998). Testing the expectations hypothesis of the term structure using instrumental variables. International Journal of Finance and Economics, 3(4), 321-325.

[10] Easterly, W. and Rebelo, S. (1993), "Fiscal policy and economic growth: an empirical investigation", Journal of Monetary Economics, 32, 417-58.

[11] Guisan, M. C., 2004. "A Comparison of Causality Tests Applied to the Bilateral Relationship between Consumption and GDP in the USA and Mexico", International Journal of Applied Econometrics and Quantitative Studies, Vol. 1, pp. 115-130

[12] Frankel, J.A. and D. Romer (1999) . “Does Trade Cause Growth?” American Economic Review 89 (1999): 379399.

[13] Florin-Marius Pavelescu (2008). Interdependences between gross capital formation, economic growth and external equilibrium in the context of the European Union enlargement în Romanian Economic Journal (Revista Română de Economie) nr. 2/2008

[14] Ighodaro, C. \& Oriakhi, D (2010). Does The Relationship Between Government Expenditure And Economic Growth Follow Wagner's Law In Nigeria?, Annals of the University of Petroşani, Economics, 10(2), 2010, 185 198.

[15] Jhingan, M.L. (2006). Economic Development, New Delhi, Vrinda Publications (P) Ltd, p.162

[16] Khan, M, and Kumar, M. (1997) Public and private investment and the growth process in developing countries, Oxford Bulletin of Economics and Statistics, 59, 69-88

[17] Grier, K. and G. Tullock, (1989). "An empirical analysis of cross-national economic growth, 1951-1980", Journal of Monetary Economics 87, 225-252.

[18] Levine, R and Renelt, D. (1992) A sensitivity analysis of cross-country growth regressions, American Economic Review, 82, 942-963.

[19] Mushtaq, M., Nazir, R., Bashir, I (2014). Panel Cointegration Analysis of Government Spending, Exports, Imports and Economic Growth, International Review of Research in Emerging Markets and the Global Economy, Vol: 1 Issue 2.

[20] Nurudeen, A. \& Usman, A (2010). Government Expenditure And Economic Growth In Nigeria, 1970-2008: A Disaggregated Analysis, Business and Economics Journal, Volume 2010: BEJ-4.

[21] Nguyễn Thị Mỹ Linh (2016). Trade Openness And Economic Growth In Countries Participating In TPP Agreement: Recommendations For Vietnam. Tạp chí Công Thương - Bộ Công Thương; (23-29). 
[22] OECD (2009), National Accounts of OECD Countries 2009, Volume I, Main Aggregates, OECD Publishing, http://dx.doi.org/10.1787/na_vol_1-2009-en-fr

[23] Okoro, S (2013). Government Spending and Economic Growth in Nigeria (1980-2011), Global Journal of Management and Business Research Economics and Commerce, Volume 13 Issue 5 Version 1.0.

[24] Phạm Thế Anh (2008). Phân tích cơ cấu chi tiêu chính phủ và tăng trưởng kinh tế ở Việt Nam. Tạp chí Nghiên cứu kinh tế, số $363,2008$.

[25] Phạm Thế Anh (2008). Chi tiêu chính phủ và tăng trưởng kinh tế: khảo sát lý luận tổng quan. Tạp chí Nghiên cứu kinh tế, số $365,2008$.

[26] Plossner, C. (1992) The search for growth in policies for long-run economic growth, Federal Reserve Bank of Kansas City, Kansas City, MO

[27] Prabirjit, S. (2007): “Trade Openness and Growth: Is there any link?” Munich Personal RePEC Archive, Paper No. 4997. http://mpra.ub.uni-muenchen.de/4997

[28] Robert J. Barr (1990). Government Spending in a Simple Model of Endogeneous Growth. Journal of Political Economy 98(S5): 103-125.

[29] Sachs, J., and A. Warner. (1995). Economic reform and the process of global integration. Brookings Paperson Economic Activity 1995(1):1-118.

[30] Yasin, M (2013). Public Spending and Economic Growth: Empirical Investigation of Sub-Saharan Africa, Morehead State University, Southwestern Economic Review.

Ngày nhận bài: 09/09/2017

Ngày chấp nhận đăng: 08/02/2018 